\title{
Notice of Retraction: Therapeutic Effects of Umbilical Cord Blood Derived Mesenchymal Stem Cell-Conditioned Medium on Pulmonary Arterial Hypertension in Rats
}

\author{
Jae Chul Lee ${ }^{1,2,3}$. Choong Ik Cha ${ }^{3}$. Dong-Sik Kim² · Soo Young Choe ${ }^{1}$ \\ 'Department of Biology, School of Life Sciences, Chungbuk National University, Cheongju; \\ 2Department of Surgery, Brain Korea 21 PLUS Project for Medical Sciences and HBP Surgery and Liver Transplantation, Korea University College of Medicine, Seoul; \\ ${ }^{3}$ Department of Anatomy, Seoul National University College of Medicine, Seoul, Korea
}

J Pathol Transl Med 2015; 49(6): 472-480. http://dx.doi.org/10.4132/jptm.2015.09.11

Editors, Journal of Pathology and Translational Medicine

Several issues related to the authorship, research materials, and protocols have been raised with the above article published in the Journal of Pathology and Translational Medicine (2015; 49: 472-80). ${ }^{1}$ The first author of the article has duely admitted that he is mainly responsible for the misconduct. Thus, the editors and publication board of the JPTM have decided to retract the article.

\section{REFERENCE}

1. Lee JC, Cha CI, Kim DS, Choe SY. Therapeutic effects of umbilical cord blood derived mesenchymal stem cell-conditioned medium on pulmonary arterial hypertension in rats. J Pathol Transl Med 2015; 49: 472-80. 